\title{
Detection of hepatitis B virus core antigen by phage display mediated TaqMan real- time immuno-PCR
}

\begin{abstract}
The core antigen ( $\mathrm{HBcAg}$ ) of hepatitis B virus (HBV) is one of the markers for the identification of the viral infection. The main purpose of this study was to develop a TaqMan real-time detection assay based on the concept of phage display mediated immuno-PCR (PDIPCR) for the detection of HBcAg. PD-IPCR combines the advantages of immuno-PCR (IPCR) and phage display technology. IPCR integrates the versatility of enzyme-linked immunosorbent assay (ELISA) with the sensitivity and signal generation power of PCR. Whereas, phage display technology exploits the physical association between the displayed peptide and the encoding DNA within the same phage particle. In this study, a constrained peptide displayed on the surface of an M13 recombinant bacteriophage that interacts tightly with HBcAg was applied as a diagnostic reagent in IPCR. The phage displayed peptide and its encoding DNA can be used to replace monoclonal antibody (mAb) and chemically bound DNA, respectively. This method is able to detect as low as $10 \mathrm{ng}$ of $\mathrm{HBcAg}$ with $108 \mathrm{pfu} / \mathrm{ml}$ of the recombinant phage which is about 10,000 times more sensitive than the phage-ELISA. The PD-IPCR provides an alternative means for the detection of $\mathrm{HBcAg}$ in human serum samples.
\end{abstract}

Keyword: TaqMan real-time PCR; Phage display mediated immuno-PCR; Hepatitis B core antigen; Phage display technology; Recombinant bacteriophage 